International Journal of Pure and Applied Mathematics

Volume 105 No. $1 \quad 2015,19-25$

ISSN: 1311-8080 (printed version); ISSN: 1314-3395 (on-line version)

url: http://www.ijpam.eu

doi: http://dx.doi.org/10.12732/ijpam.v105i1.3

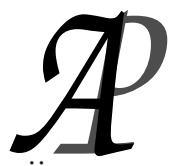

ijpam.eu

\title{
ABEL'S CLASSES OF EQUATIONS WITH CLOSED FORM SOLUTION
}

\author{
Cesar A. Gómez S. \\ Departamento de Matemáticas \\ Universidad Nacional de Colombia \\ Bogotá, Kra. 30 No. 45-03, of. 209-404, COLOMBIA
}

\begin{abstract}
We present some Abel equations of first kind with solution in closed form. By using an adequate transformation the initial Abel equation is converted to a standard equation from which some conditions for its integrability can be observed. Several relations between the coefficients of the initial Abel equation are derived with the aim to obtain its solution in closed form.
\end{abstract}

AMS Subject Classification: $34 \mathrm{~A} 34$

Key Words: Abel equation of first kind, closed form solution, implicit solution, analytical method

\section{Introduction}

One of the most important nonlinear first order ordinary differential equation is given by the so called Abel equation of first kind which have the form

$$
\frac{d y}{d x}=p(x) y^{3}(x)+q(x) y^{2}(x)+r(x) y(x)+s(x) .
$$

Received: June 13, 2015

(c) 2015 Academic Publications, Ltd. url: www.acadpubl.eu 
It is clear that (1) is the natural generalization of the very important Riccati equation

$$
\frac{d y}{d x}=p(x) y^{2}(x)+q(x) y(x)+r(x)
$$

which appear in many fields of the pure and applied mathematics. The equation (1) have been intensively studied from of point of view of its analytic solutions as well as of its applications in many fields of the applied physics $[1],[2],[3],[4],[5],[6],[7]$. As in the case of Eq.(2) [8], the general solution of Eq.(1) cannot be obtain in elementary form, however several transformations, relations between its coefficients and additional conditions have been presented with the aim to reduce the equation to one which can be solved directly and in this way to obtain solutions to initial equation [1],[2],[3],[4],[5]. In this work, a class of Abel equations of first kind with analytic solution are studied. More exactly, we consider the equation

$$
\frac{d y}{d x}=p^{2}(x) y^{3}(x)+q(x) y^{2}(x)+r(x) y(x)+s(x),
$$

where $p(x), q(x), r(x)$ and $s(x)$ are continuous functions defined on the real interval $[a, b]$ and $p(x) \neq 0$ for $x \in[a, b]$ and we obtain some conditions under which (3) can be solved in direct form.

\section{Special Abel Equations with Closed Form Solution}

We consider the Abel equation (3). Whit the change of variable

$$
y(x)=\frac{1}{p(x)}\left(u(x)-\frac{q(x)}{3 p(x)}\right)
$$

Eq.(3) is transformed into equation

$$
\frac{d u}{d x}=u(x)^{3}+A(x) u(x)+B(x),
$$

where

$$
A(x)=\frac{p^{\prime}(x)}{p(x)}+r(x)-\frac{q^{2}(x)}{3 p(x)^{2}}
$$

and

$$
B(x)=\frac{1}{27 p(x)^{3}}\left\{\begin{array}{l}
27 s(x) p(x)^{4}-9 q(x) r(x) p(x)^{2}+ \\
9 q^{\prime}(x) p(x)^{2}-18 q(x) p^{\prime}(x) p(x)+2 q(x)^{3} .
\end{array}\right.
$$


Now, with the help of the substitution

$$
u(x)=z(x) e^{\int A(x) d x},
$$

Eq.(5) reduces to

$$
\frac{d z}{d x}=z(x)^{3} e^{2} \int A(x) d x+\frac{B(x)}{e^{\int A(x) d x}} .
$$

We can see two special cases in which the Eq.(3) can be integrate.

Theorem 1. If the coefficients of Eq.(3) satisfies the relation

$$
\frac{27 s(x) p(x)^{4}-9 q(x) r(x) p(x)^{2}+9 q^{\prime}(x) p(x)^{2}-18 q(x) p^{\prime}(x) p(x)+2 q(x)^{3}}{27 p(x)^{3}}=0
$$

then the general solution of (3) is given by

$$
y(x)=\frac{1}{p(x)}\left( \pm \frac{e^{\int A(x) d x}}{\sqrt{-2 \int e^{2} \int A(x) d x}+k}-\frac{q(x)}{3 p(x)}\right),
$$

being $k$ an arbitrary constant.

Proof. The condition is equivalent to say that $B(x)=0$, so that (9) is a separable variables equation which solution is

$$
z(x)= \pm \frac{1}{\sqrt{-2 \int e^{2} \int A(x) d x}+k} .
$$

Now, taking into account (8) and (4) we obtain (11).

Example 1: A particular case of theorem is the equation

$$
\frac{d y}{d x}=\left\{\begin{array}{l}
p^{2}(x) y^{3}(x)+q(x) y^{2}(x)+r(x) y(x)+ \\
\frac{-2 q(x)^{3}+9 p(x)^{2} r(x) q(x)+18 p(x) p^{\prime}(x) q(x)-9 p(x)^{2} q^{\prime}(x)}{27 p(x)^{4}}
\end{array}\right.
$$


with $p(x), q(x)$ and $r(x)$ arbitrary functions. In we take $p(x)=x, q(x)=$ $\sqrt{3} x$, and $r=1$ we can obtain the solution of equation $y^{\prime}(x)=x^{2} y(x)^{3}+$ $\sqrt{3} x y(x)^{2}+y(x)+\frac{\sqrt{3} x+3 \sqrt{3}}{9 x^{2}}$. In fact, we have $A(x)=\frac{1}{x}$ so that by (11) $y(x)=$ $\frac{1}{x}\left( \pm \frac{x}{-\frac{2}{3} x^{3}+k}-\frac{\sqrt{3}}{3}\right)$ is the solution.

Example 2: If additionally we have $A(x)=0$ in the theorem, then the equation (3) have solution in closed form. The following equation is a particular case of this situation:

$$
\frac{d y}{d x}=\left\{\begin{array}{l}
p^{2}(x) y^{3}(x)+q(x) y^{2}(x)+\left(\frac{q^{2}(x)}{3 p(x)^{2}}-\frac{p^{\prime}(x)}{p(x)}\right) y(x)+ \\
\frac{q(x)^{3}+9 p(x) p^{\prime}(x) q(x)-9 p(x)^{2} q^{\prime}(x)}{27 p(x)^{4}}
\end{array}\right.
$$

so that the solution of $\frac{d y}{d x}=x^{2} y(x)^{3}+\sqrt{3} x y(x)^{2}+\left(1-\frac{1}{x}\right) y(x)+\frac{\sqrt{3}}{9 x}$ can be obtain using (11). In this case we have the solution $y(x)=\frac{1}{x}\left( \pm \frac{1}{\sqrt{-2 x+k}}-\frac{\sqrt{3}}{3}\right)$.

Example 3: Other particular case of the theorem is given by equation

$$
\frac{d y}{d x}=\left\{\begin{array}{l}
p^{2}(x) y^{3}(x)+q(x) y^{2}(x)+ \\
\left(\frac{27 s(x) p(x)^{4}+9 q^{\prime}(x) p(x)^{2}-18 q(x) p^{\prime}(x) p(x)+2 q(x)^{3}}{9 p(x)^{2} q(x)}\right) y(x)+s(x),
\end{array}\right.
$$

being $p(x), q(x)$ and $s(x)$ arbitrary functions.

Theorem 2. If the coefficients of (3) satisfies the relations

$$
\left\{\begin{array}{l}
\frac{2 q(x)^{3}}{27 p(x)^{3}}-\frac{q(x) r(x)}{3 p(x)}+p(x) s(x)-\frac{2 q(x) p^{\prime}(x)}{3 p(x)^{2}}+\frac{q^{\prime}(x)}{3 p(x)}-27 p(x)^{6} k^{3}=0, \\
3 p(x)^{2} r(x)-3 p(x) p^{\prime}(x)-q(x)^{2}=0
\end{array}\right.
$$

being $k$ an arbitrary constant, then (3) can be solve exactly.

Proof. In the first equation of (16), the case $k=0$ is equivalent to $B(x)=$ 0 and therefore the equation can be solved using the theory of the previous section. We consider the case $k \neq 0$. The second condition of (16) is equivalent to $A(x)=\frac{6 p(x) p^{\prime}(x)}{3 p(x)^{2}}$ and therefore $(9)$ converts to

$$
\frac{d z}{d x}=9 z(x)^{3} p(x)^{4}+\frac{B(x)}{3 p(x)^{2}} .
$$

Now, using the change of variable

$$
\xi=9 p(x)^{4}
$$


then (17) take the form

$$
\frac{d z}{d \xi}=z(\xi)^{3}+\frac{B(\xi)}{27 p(\xi)^{6}}
$$

The first condition of (16) say us that $\frac{B(\xi)}{27 p(\xi)^{6}}=k^{3}$ and therefore the solution of (19) is given by

$$
\frac{2 \sqrt{3} \tan ^{-1}\left(\frac{2 z(\xi)-k}{\sqrt{3} k}\right)+2 \log (k+z(\xi))-\log \left(k^{2}-z(\xi) k+z(\xi)^{2}\right)}{6 k^{2}}=\xi+C,
$$

with $C$ the integration constant. Finally, taking into account (20),(18), (8) and (4)the solution of (3) is obtained.

Example 4: The following are Abel equations of the form (3) which satisfies the conditions of this last theorem:

1.

$$
\frac{d y}{d x}=h(x)\left(1-\frac{2}{3} \int^{x} \frac{q(\nu)}{h(\nu)} d \nu\right) y(x)^{3}+q(x) y(x)^{2}+r(x) y(x)+s(x),
$$

where $h(x)=e^{\mathbf{R}_{x}} 2 r(t) d t$,

$s(x)=\frac{729 k^{3} h(x)^{9 / 2}+9 q(x) r(x) h(x)-9 q^{\prime}(x) h(x)-2 q(x)^{3}+9 q(x) h^{\prime}(x)}{27 h(x)^{2}}$.

2 .

$$
\frac{d y}{d x}=p^{2}(x) y^{3}(x)+\left(\sqrt{3 p(x)\left(p(x) r(x)-p^{\prime}(x)\right)}\right) y^{2}(x)+r(x) y(x)+s(x),
$$

where

$$
s(x)=\frac{243 k^{3} p(x)^{8}+\left(\sqrt{3} r(x) \sqrt{p(x)\left(p(x) r(x)-p^{\prime}(x)\right)}-3 q^{\prime}(x)\right) p(x)+8 \sqrt{3} \sqrt{p(x)\left(p(x) r(x)-p^{\prime}(x)\right)} p^{\prime}(x)}{9 p(x)^{3}} .
$$

3.

$$
\frac{d y}{d x}=p^{2}(x) y^{3}(x)+q(x) y^{2}(x)+\left(\frac{q(x)^{2}+3 p(x) p^{\prime}(x)}{3 p(x)^{2}}\right) y(x)+s(x),
$$

where

$s(x)=\frac{q(x)^{3}+9 p(x)\left(81 k^{3} p(x)^{8}-q^{\prime}(x) p(x)+3 q(x) p^{\prime}(x)\right)}{27 p(x)^{4}}$.

The following Abel equation is a particular case of the first model:

$$
\frac{d y}{d x}=\left(x^{2}-\frac{2}{3} x^{3}\right) y(x)^{3}+x^{2} y(x)^{2}+\frac{1}{x} y(x)+\frac{729 k^{3} x^{6}-2 x^{3}+9}{27 x} .
$$




\section{Conclusions}

We have presented some Abel equations of first kind which can be solved in closed form. This equations were obtained after reduce a special Abel equation to a standard equation. Several conditions for its integrability were observed. However, it is possible to derive other conditions to obtain Abel's classes of equations with exact analytic solution. More exactly if we use the transformation $\xi=\int e e^{\int 2 A(x) d x}$ then (9) reduces to

$$
\frac{d z}{d \xi}=z(\xi)^{3}+\frac{B(\xi)}{F(\xi)}
$$

where $F(x)=e^{\int 3 A(x) d x}$. If the coefficients of (3) satisfies the condition

$$
\left\{\begin{array}{l}
81 r(x) s(x) p(x)^{6}-27 s^{\prime}(x) p(x)^{6}+54 s(x) p^{\prime}(x) p(x)^{5} \\
-27 q(x) r(x)^{2} p(x)^{4}-27 q(x)^{2} s(x) p(x)^{4}+36 r(x) q^{\prime}(x) p(x)^{4} \\
+9 q(x) r^{\prime}(x) p(x)^{4}-9 q^{\prime \prime}(x) p(x)^{4}-90 q(x) r(x) p^{\prime}(x) p(x)^{3}+ \\
54 p^{\prime}(x) q^{\prime}(x) p(x)^{3}+18 q(x) p^{\prime \prime}(x) p(x)^{3} \\
-90 q(x) p^{\prime}(x)^{2} p(x)^{2}+15 q(x)^{3} r(x) p(x)^{2}-15 q(x)^{2} q^{\prime}(x) p(x)^{2}+ \\
30 q(x)^{3} p^{\prime}(x) p(x)-2 q(x)^{5}=0,
\end{array}\right.
$$

then (21) converts to separable variables equation $\frac{d z}{d \xi}=z(\xi)^{3}+1$.

\section{References}

[1] L. Bougoffa, New exact general solutions of Abel equation of the second kind, Appl. Math. and Comp., 216 (2010), 689-691. doi:10.1016/j.amc.2010.01.114

[2] D.E. Panayotounakos, Exact analytic solutions of unsolvable classes of first and second order nonlinear ODEs (Part I. Abel's Equations), Applied Mathematics Letters, 18 (2005), 155-162. doi:10.1016/j.aml.2004.09.004

[3] M.K. Mak and T. Harko, New Method for Generating General Solution of Abel Differential Equation, An Int. J. Computer and Mathematics with Applications, 43 (2002), 91-94. doi:10.1016/S0898-1221(01)00274-7 
[4] J.M. Olm, X. Ros-Oton and T.M. Seara, Periodic solutions with nonconstant sign in Abel equations of the second kind, J. of Mathematical Analysis and Applications, 381 (2011), 582-589. doi:10.1016/j.jmaa.2011.02.084

[5] A.D. Polyanin and V.F. Zaitsev, Handbook of Exact Solutions for Ordinary Differential Equations, CRC Press, New York (1999).

[6] D.E. Panayotounakos, A.B. Sotiropoulou, N.B. Sotiropoulos and M. Manios, Exact analytic solutions of the porous media and the gas pressure diffusion ODEs in non-linear mechanics, Int. J. of NON-LINEAR MECHANICS, 42 (2007), 157-163. doi:10.1016/j.ijnonlinmec.2006.10.018

[7] A.B. Sotiropoulou and D.E. Panayotounakos, On the reduction of some second-order nonlinear ODEs in physics and mechanics to first-order nonlinear integrodiferential and Abel's classes of equations, Theoretical and appl. fracture Mechanics, 40 (2003), 255-270. doi:10.1016/j.tafmec.2003.09.001

[8] C.A. Gomez and A. Salas, Special symmetries to standard Riccati equations and applications, Appl. Math. and Comp., 216 No. 10 (2010), 30893096. doi:10.1016/j.amc.2010.04.039 
